\title{
Comparison of Algorithms for Searching Shortest Path and Implementations for the Searching Routing System via Web Services
}

\author{
Pattama Charoenporn ${ }^{+}$ \\ ${ }^{1}$ Department of Computer Science, Faculty of Science \\ King Mongkut's Institute of Technology Ladkrabang \\ Bangkok, Thailand
}

\begin{abstract}
The searching routing system is part of logistics systems. There are many algorithms to solve the detail of confuse routing, e.g., routing of transport networks or order picking areas. The numbers of relevant nodes in routing have more than 10,000 nodes. Then many algorithms should be finding the shortest path from start node to destination. In this paper, we choose 3 algorithms compose of Floyd-Warshall's Algorithm, Dijkstra's Algorithm and saving algorithm with google map api to compare the best algorithm to searching shortest path and then choose the best algorithm to implement searching routing system via web services. For the results, Floyd-Warshall's Algorithm and Dijkstra's Algorithm give same results but the results from saving algorithm with google map api is the best algorithm for searching and manage the transport route to the point of destination especially in the real time. Then, this research will use saving algorithm for solving the ordering of transportation and use Google maps API for set direction. Google maps API can advise the shortest path and mark start node, end node for user. Saving algorithm find the best route and calculate the result from equation.
\end{abstract}

Keywords: floyd-warshall's algorithm, dijkstra's algorithm, saving algorithm, Google maps API, shortest path.

\section{Introduction}

One of the best problems in logistics systems is the routing and scheduling of vehicles through a set of nodes for routing system. Many organizations bring the vehicle routing problem (VRP) to manage a set of minimum-cost vehicle routes, begin node and end node, for searching shortest routes. But some of problems occur in VRP, e.g., some algorithm doesn't support all searching routing system or doesn't calculate the correctly shortest path. Because sometime many transportations were transport daily and a truck doesn't follow the route that generate by searching routing system. The problem is occurring in the real world. This research presents the saving algorithm with google map API. And test the prototype by compare results with 3 algorithms. In order to choose the best way for solve problems.

For searching routing system, it is a variety of logistics system where is set up to analyse systems in which the routing of personnel, e.g., workers or order pickers, and vehicles is a relevant part of the modelled process. Some task in searching routing system is find the shortest path from a begin position to end destination (within a graph of nodes). For this problem, some of algorithms are created. Several searching routing systems offer an automatic routing of vehicles, such that a vehicle takes the shortest path from the begin position (node) to end destination node automatically.

We implement searching routing system via web services with respect to performance of automatically routing vehicles in real time from a begin position to end destination on the shortest path within a transport

\footnotetext{
+ Corresponding author. Tel.: ++662-329-8400; fax: +662329-8412.

E-mail address: pattama.ch@kmitl.ac.th
} 
network. And we test this searching routing system to user, all of feedback show that this searching routing system can useful and can searching routing in real time.

\section{Shortest Path Algorithms}

\subsection{Floyd-Warshall's Algorithm}

This algorithm was developed independently from each other by Floyd and Warshall [1]. Instead of computing a path from a given start node to all other nodes (or a single destination node), all shortest paths, e.g., from each node to all others, are computed within a single loop. As a result we obtain a matrix Dist, where Dist $[i, j]$ denotes the distance from node i to node $j$. Furthermore, a matrix Next can be computed where Next $[i, j]$ represents the successor of node $i$ on the shortest path from node $i$ to node $j$

for all nodes i of $\mathrm{N}$ do

for all nodes $\mathrm{j}$ of $\mathrm{N}$ do

if there is an edge from $\mathrm{i}$ to $\mathrm{j}$ then $\operatorname{Dist}[\mathrm{i}, \mathrm{j}]:=\mathrm{d}(\mathrm{i}, \mathrm{j})$ else Dist $[\mathrm{i}, \mathrm{j}]:=\infty$

for all nodes $\mathrm{i}$ of $\mathrm{N}$ do for all nodes $\mathrm{j}$ of $\mathrm{N}$ do

for all nodes $\mathrm{k}$ of $\mathrm{N}$ do if $\operatorname{Dist}[\mathrm{j}, \mathrm{i}]+\operatorname{Dist}[\mathrm{i}, \mathrm{k}]<\operatorname{Dist}[\mathrm{j}, \mathrm{k}]$ then $\operatorname{Dist}[\mathrm{j}, \mathrm{k}]:=\operatorname{Dist}[\mathrm{j}, \mathrm{i}]+\operatorname{Dist}[\mathrm{i}, \mathrm{k}] ; \mathrm{Next}[\mathrm{j}, \mathrm{k}]:=\mathrm{i}$;

\subsection{Dijkstra's Algorithm}

This algorithm delivers the shortest path from a given node $i$ to a single destination node or all other nodes within a graph with nonnegative edge path costs [2]. The idea is to set up a set of unvisited nodes and the tentative distance from node $\mathrm{i}$ to all other nodes $\mathrm{j}$, denoted as Dist $[\mathrm{j}]$. Furthermore, a list Prev[j] stores the previous node on the path from node $i$ to node $j$. All distances are initially set to infinity. The start node $i$ is the initial current node. For the current node all of its unvisited neighbours (connected by an edge) are considered next and the tentative distances to these neighbours are calculated. After all neighbour nodes of the current node have been considered, the current node is deleted from the set of unvisited nodes. The next node selected as the current node is the one with the shortest distance to node i (i.e., the node which has the minimum value in Dist[j]). The algorithm terminates once the destination node has been deleted from the set of unvisited nodes, or once all nodes have been considered and the set of unvisited nodes is empty.

\subsection{Google map api}

Google Maps API [5] as a service tool from Google application for development a web application and mobile application (Android, iOS). Many applications can access to the google map and services via internet. Google Map API composes of 6 functions: Google Maps Geocoding API, Google Maps Geolocation API, Google Places API, Google Maps Distance Matrix API, Google Maps Directions API, Google Maps Roads API

\subsection{Saving Algorithm [3]}

The method is well known for managing the transportation. It can search the best route for vehicle that will be sending from multiple customers. The method is known heuristic method, which solves the problem of vehicle routing (VRP - Vehicle Routing Problem), is Clarke-Wright's method. Solving the role of routing by Clarke-Wright's method is carried out by gradual steps. Firstly, the least preferred solution, which is then improved by each gradual step, is found. And then defined conditions can be monitored and controlled by gradual steps. The whole procedure of solving the role of routing is taken over according to [4] as follows: The role of routing is generally formulated on the transport network of $\mathrm{S}-(\mathrm{V} ; \mathrm{H})$, where $\mathrm{V}$ is the set of nodes of the network and $\mathrm{H}$ is the set of edges connecting these nodes. Node $\mathrm{V} 0$ represents a wholesale warehouse of a given transport network, nodes $\mathrm{V}_{1}, \ldots, \mathrm{V}_{\mathrm{N}}$ are delivery points (points requiring manual). Each delivery point has a certain requirement for the transportation.

\section{Comparison of Difference Routing Algorithms}

We compare three different approaches for finding shortest paths in road networks can be found in figure one. The experiment set up in route of suvarnabhumi airport in Thailand. We set two routes and separate two ways. (1: denote by green box and 2: denote by yellow box). We test each shortest path and compare results 
from three algorithms (Floyd-Warshall's Algorithm, Dijkstra's Algorithm, saving algorithm with google map api).
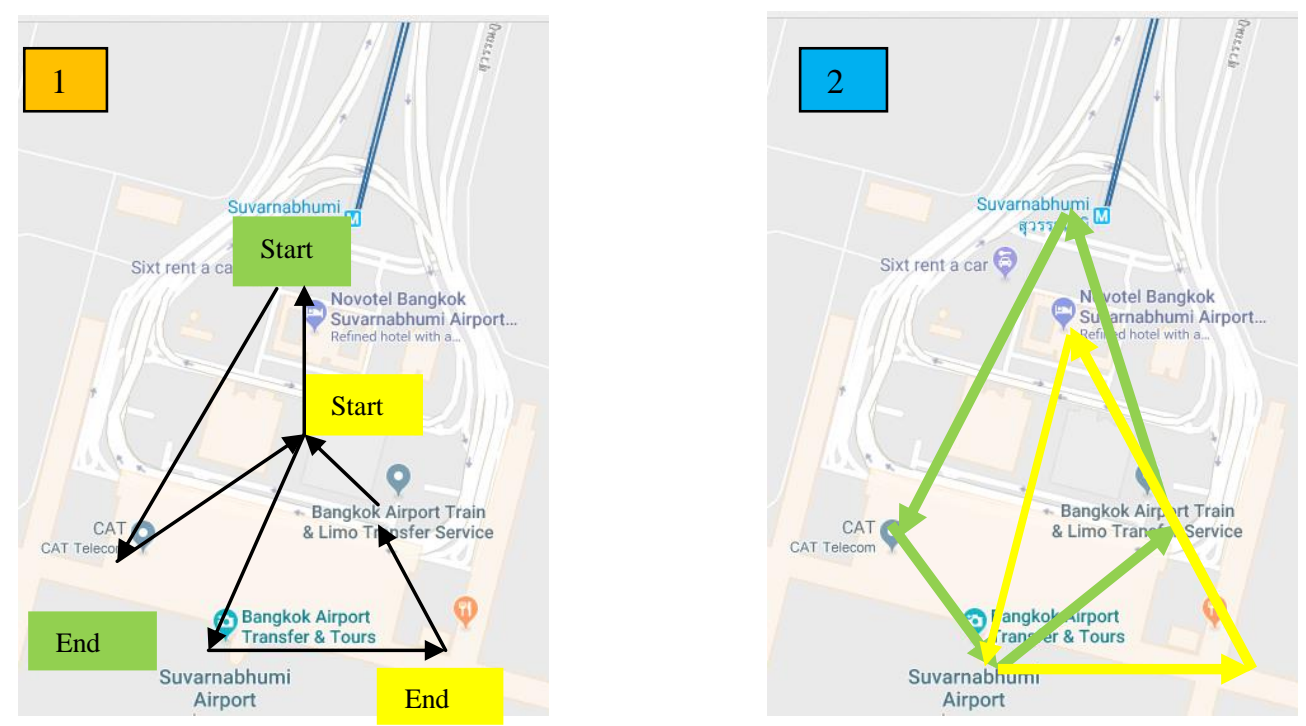

Fig. 1: Route of suvarnabhumi airport in Thailand.

In figure one, shows a vehicle routing problem with two vehicles (denote by yellow and green boxes). They start at the airport and return to end node after serving the customers. Obviously, all of routes are not the optimal solution for shortest path. The number two illustrate adaptation process. In picture number two represents the solution after the removal by the destroy point. The tours remain plenary, but some customers are not served any more. Then, we consider two sets of test problems in order to compare three algorithms. First, select start node from map $\left(\mathrm{D}_{0}\right)$ commodities should be distributed end point of customer's. We assume that $\mathrm{C}_{\mathrm{i}}$ is route of travelling in map.

$$
\mathrm{C}_{\mathrm{i}} \quad \text { where } \mathrm{I}=1,2, \ldots, \mathrm{n} .
$$

We set latitude and longitude follow google map api for test three algorithms. The locations are given in Table I by their coordinates.

Table 1: Location

\begin{tabular}{|c|c|c|c|c|c|}
\hline & $\mathbf{D}_{\mathbf{1}}$ & $\mathbf{C}_{\mathbf{2}}$ & $\mathbf{C}_{\mathbf{3}}$ & $\mathbf{C}_{\mathbf{4}}$ & $\mathbf{C}_{\mathbf{5}}$ \\
\hline $\mathbf{X}_{\mathbf{i}}$ & 13.728 & 13.721 & 13.699 & 13.815 & 13.72 \\
\hline $\mathbf{Y}_{\mathbf{i}}$ & 100.78 & 100.79 & 100.65 & 100.74 & 100.7 \\
\hline $\mathbf{Q}_{\mathbf{i}}$ & 0 & 38 & 57 & 54 & 214 \\
\hline
\end{tabular}

After that, we replace input in algorithms and test it. The results will be separate according two routes. We are setting the same of conditions e.g. weight must have $500 \mathrm{Kg} / \mathrm{truck}$, motor fuel for $15 \mathrm{~km} / \mathrm{L}$ and must spending for the diesel for 27.19 Baht. The results shown in table II.

Table 2: The result

\begin{tabular}{|l|l|l|c|l|}
\hline \multirow{2}{*}{ Algorithms } & \multicolumn{4}{|c|}{ Results } \\
\cline { 2 - 5 } & Distance & Time & Count & Costs \\
\hline Floyd-Warshall's Algorithm & $205 \mathrm{~km}$ & $5 \mathrm{~h} .44 \mathrm{~m}$ & 3 & 371.596 Baht \\
\hline Saving Algorithm & $209.2 \mathrm{~km}$ & $5 \mathrm{~h} .50 \mathrm{~m}$. & 3 & 391.898 Baht \\
\hline Dijkstra's Algorithm & $195 \mathrm{~km}$ & $5 \mathrm{~h} .38 \mathrm{~m}$ & 3 & 368.596 Baht \\
\hline
\end{tabular}

The results shown that dijkstra's algorithm is the best algorithm for searching routing than the others. But this algorithm doesn't help when user want to change route at real time then we choose saving algorithm 
because it can coordinate with google map api. When we modify some variables in saving algorithm and set the latitude $\left(\mathrm{X}_{\mathrm{i}}\right)$ and longitude $\left(\mathrm{Y}_{\mathrm{i}}\right)$. To calculate distance and set all of distance metric with google distance matrix api.The results shown in Table III.

Table 3: Distance metric

\begin{tabular}{|l|c|c|c|c|c|}
\hline & $\mathbf{D}_{\mathbf{1}}$ & $\mathbf{C}_{\mathbf{2}}$ & $\mathbf{C}_{\mathbf{3}}$ & $\mathbf{C}_{\mathbf{4}}$ & $\mathbf{C}_{\mathbf{5}}$ \\
\hline $\mathbf{C}_{\mathbf{2}}$ & 2.1 & 0 & & & \\
\hline $\mathbf{C}_{\mathbf{3}}$ & 21.4 & 21 & 0 & & \\
\hline $\mathbf{C}_{\mathbf{4}}$ & 16.2 & 15 & 31.5 & 0 & \\
\hline $\mathbf{C}_{\mathbf{5}}$ & 11.9 & 11.5 & 13.3 & 15.7 & 0 \\
\hline
\end{tabular}

And bring value of D1 from table III to calculate in saving algorithm. The results show in table IV

Table 4: Saving costs

\begin{tabular}{|c|c|c|c|c|c|c|c|}
\hline No. & Saving & \multicolumn{2}{|c|}{$\mathbf{C}_{\mathbf{i}}$} & No. & Saving & \multicolumn{2}{|c|}{$\mathbf{C}_{\mathbf{i}}$} \\
\hline $\mathbf{1}$ & 41.3 & $\mathrm{C}_{6}$ & $\mathrm{C}_{8}$ & $\mathbf{6}$ & 20.1 & $\mathrm{C}_{5}$ & $\mathrm{C}_{6}$ \\
\hline $\mathbf{2}$ & 36.6 & $\mathrm{C}_{6}$ & $\mathrm{C}_{7}$ & $\mathbf{7}$ & 20 & $\mathrm{C}_{3}$ & $\mathrm{C}_{5}$ \\
\hline $\mathbf{3}$ & 33.9 & $\mathrm{C}_{4}$ & $\mathrm{C}_{6}$ & $\mathbf{8}$ & 19.3 & $\mathrm{C}_{3}$ & $\mathrm{C}_{9}$ \\
\hline $\mathbf{4}$ & 31.5 & $\mathrm{C}_{7}$ & $\mathrm{C}_{8}$ & $\mathbf{9}$ & 14 & $\mathrm{C}_{3}$ & $\mathrm{C}_{8}$ \\
\hline $\mathbf{5}$ & 30.8 & $\mathrm{C}_{4}$ & $\mathrm{C}_{8}$ & $\mathbf{1 0}$ & 12.4 & $\mathrm{C}_{4}$ & $\mathrm{C}_{5}$ \\
\hline
\end{tabular}

Select the maximum route under citeria weight of a car, weight of products and calculate again.

Table 5: Route

\begin{tabular}{|c|c|c|}
\hline Route & Load & Total distance \\
\hline D1-C6-C8-D1 & 490 & \multirow{2}{*}{216.2} \\
\hline D1-C2-C4-C7-C3-D1 & 349 & \\
\hline D1-C5-C9-D1 & 234 & \\
\hline
\end{tabular}

Finally, we customize saving algorithm by used google map api to solve problem. In this case, the algorithm improved and denote in below.

Input: $\mathrm{X}_{\mathrm{i}}$; A latitude of each location for transport. $\mathrm{Y}_{\mathrm{i}}$; A longitude of each location for transport.

$\mathrm{Q}_{\mathrm{i}}$; The mass of products for transport.

The number of vehicle could be able to transport with a maximum mass.

1: for all $x_{i}$ and $y_{i}$ do

2: $\quad$ distance $\left[x_{i}\right]\left[y_{i}\right]=$ getDistanceAPIs $\left(x_{i}, y_{i}\right)$

3:end for

4: for all distance $\left[x_{i}\right]\left[y_{i}\right]$ do

5: $\quad \operatorname{saving}\left[x_{i}\right]\left[y_{i}\right]=\left(\operatorname{distance}\left[x_{i}\right][1]+\operatorname{distance}\left[y_{i}\right][1]\right)-\operatorname{distance}\left[x_{i}\right]\left[y_{i}\right]$

//use Saving Algorithm

6: end for

7: for all saving $\left[x_{i}\right]\left[y_{i}\right] d o$

8: $\quad$ if saving $\left[x_{i}\right]\left[y_{i}\right]>\max$

9: $\quad \max [i]=\operatorname{saving}\left[x_{i}\right]\left[y_{i}\right]$ end if

10: end for

11: for all truck do

// assign order to each trucks

12: for all $\max [i] \mathrm{do}$

13: assignRoute() endfor

14: end for

Output: Transportation queue. 
For algorithm, if route $\mathrm{C} 2$ and $\mathrm{C} 3$ is added to the path, then the saving algorithms connecting customer and put value in the queue. The algorithm will be calculated the shortest path and set the shortest routing path for truck. For the routes derive form google maps api. when user choose the start point and destination point google maps api will calculate the distance and show the best routes of transport for each destination, after that saving algorithm will be arranging queue and send to customer again.

\section{Implement the Searching Routing System via Web Services}

After we customize saving algorithm with google map api and test algorithm completely, we create the prototype follow by service oriented architecture. The architecture composes of three parts: service provider, service register and service consumer. In this experiment we design web service in service provider only; it has three services for customer who want to use this prototype. The services compose of searching routing, detail routing, calculate expense for transport. Finally, we take this prototype to test with user approximate 400 users. They answer all of question in the opinion form. It suggests that; $80 \%$ choose very satisfy in efficiency because it can use all business, $67 \%$ choose very satisfy in learn ability. And all of them choose satisfy in prototype.

\section{Conclusion and Future Work}

These researches present how to solve the problem of the wasted route in transportation. The process for solve this problem compose of two methods: 1 . Compare method from three algorithms 2 . Implement searching routing system via web services. This results explain that saving algorithm with google maps API would be solving and manage the transport to the point of destination especially the transport in the real time, it happened under queue by ordering from algorithm. This API will calculate the best distance (shortest) and fuel fee of each time for assessment average timing to customers. Saving Algorithm could be able to solve many problems for transportation which knowing the number of vehicle, the step for packaging and weight of the products in each of vehicle. This research presents the saving algorithm for generate the best route and applying them with google maps API will get the best distance for quickly and real time to transporting. Saving algorithm is a theory that was accepted in management problem of transport vehicles and most popular in today because this theory is not complicate and easy to understand. For this research, we have tested our approach with some real data from the route in Thailand. The experimental results seem to validate the competitiveness of the 2 algorithm, since it is able to improve both other academic approaches as well as real routing plans developed by experts in the company. When we choose algorithm to searching shortest path already, we get it to a part of implement searching routing system via web services. All of user who used this prototype like and satisfy it. Finally, some of biased in opinion occur in a wide range of classical heuristics which are oriented to other optimization problems. In the future, we will find another algorithm to generate method for searching shortest path. And the prototype must be compatible in many situations for logistics system and the application must operate under other factors in completely.

\section{Reference}

[1] Hochbaum, Dorit ."Section 8.9: Floyd-Warshall algorithm for all pairs shortest paths" (PDF). Lecture Notes for IEOR 266: Graph Algorithms and Network Flows. Department of Industrial Engineering and Operations Research, University of California, Berkeley,2014.

[2] Daniel A. Spielman "Design and Analysis of Algorithm Lecture 5 Shortest path", available from: http://www.cs.yale.edu/homes/spielman/365/shortestPaths.pdf, 2017.

[3] Kampf, R., Stopka, O., Bartuska, L., Zeman, K. Circulation of vehicles as an important parameter of public transport effciency. In: Transport Means Proceedings of the 19th International Scientifc Conference on Transport Means. Kaunas (Lithuania): Kaunas University of Technology, 2015, pp. 143-146. ISSN 1822-296X

[4] Build the next generation of location experiences, available from : https://developers.google.com/maps/

[5] Wang Xing, Zhao Shu-Zhi Wright "An Improved Savings Method for Vehicle Routing Problem”, 2nd International Conference on Control Science and Systems Engineering, IEEE Publications, 2016. 